\title{
The dosimetric comparison using anisotropic analytical algorithm (AAA) and Acuros XB algorithms in the target overlapping with air cavity of pituitary carcinoma
}

\author{
Isra Israngkul-Na-Ayuthaya ${ }^{1}$, Sivalee Suriyapee ${ }^{2}$, Taweap Sanghamthum ${ }^{2}$ \\ ${ }^{1}$ Division of Therapeutic Radiology and Oncology, Department of Radiology, King Chulalongkorn Memorial Hospital, Bangkok, Thailand; ${ }^{2}$ Division \\ of Therapeutic Radiology and Oncology, Faculty of Medicine, Chulalongkorn University, Bangkok, Thailand \\ Contributions: (I) Conception and design: I Israngkul-Na-Ayuthaya; (II) Administrative support: S Suriyapee; (III) Provision of study materials or \\ patients: I Israngkul-Na-Ayuthaya; (IV) Collection and assembly of data: I Israngkul-Na-Ayuthaya; (V) Data analysis and interpretation: I Israngkul- \\ Na-Ayuthaya, T Sanghangthum; (VI) Manuscript writing: All authors; (VII) Final approval of manuscript: All authors. \\ Correspondence to: Isra Israngkul-Na-Ayuthaya. Division of Therapeutic Radiology and Oncology, Department of Radiology, King Chulalongkorn \\ Memorial Hospital, 1873 Rama IV Rd., Pathum Wan, Pathum Wan District, Bangkok 10330, Thailand. Email: linkinpond@gmail.com.
}

Background: This study was to investigate the dose variation between anisotropic analytical algorithm (AAA) and Acuros XB (AXB) calculation algorithms in heterogeneous inside planning target volume (PTV) of pituitary carcinoma.

Methods: AAA and AXB algorithms were used for dose calculation by Eclipse treatment planning system in Varian TrueBeam linear accelerator machine with 120 MLC. Twelve cases of pituitary carcinoma were planned by intensity-modulated radiotherapy (IMRT) and volumetric modulated arc therapy (VMAT) techniques. The target overlapped with the air cavity. IMRT plans were done by 9 static fields and VMAT plans were done by 2-3 arc rotation fields. The PTV was prescribed the dose of 50.4 Gy. The dosimetry was analyzed by dose to $95 \%$ and $98 \%$ of volume of PTV, maximum dose, dose coverage, and conformity index (CI) of PTV. The normal organ was analyzed by mean dose (Dmean) and maximum dose (Dmax).

Results: The dose coverage of PTV using AXB calculation was less than using AAA calculation in the air cavity region for both IMRT and VMAT. The dose coverage of PTV was any statistical significantly difference between AAA and AXB algorithms. For the normal organs, the mean and maximum dose agreed well for AAA and AXB algorithms.

Conclusions: AXB can calculate the dose more accurate than AAA for dose calculation in inhomogeneous region. AXB algorithms should be selected for dose calculation in clinical.

Keywords: Anisotropic analytical algorithm (AAA); Acuros XB (AXB); treatment planning, inhomogeneity, air cavity

Received: 14 October 2020; Accepted: 29 March 2021; Published: 30 June 2021.

doi: $10.21037 /$ tro-20-57

View this article at: http://dx.doi.org/10.21037/tro-20-57

\section{Introduction}

In radiotherapy, the prescription dose delivered to the patient must be accurate with $\pm 5 \%$ of overall (1). The treatment planning is an important system to reduce the variation of the dose to patient in the dose calculation process which depends on the dose calculation algorithms.

Eclipse treatment planning (Varian Medical Systems, Palo
Alto, CA, USA) provides two mainly algorithms to calculate dose which the one is anisotropic analytical algorithm (AAA) and the other one is Acuros $\mathrm{XB}(\mathrm{AXB})$ algorithm. The AAA which has been implemented in Eclipse TPS is one of a $3 \mathrm{D}$ pencil beam convolution-superposition algorithm. AAA model configuration is based on basic physical parameters which are determined by Monte-Carlo. This algorithm 


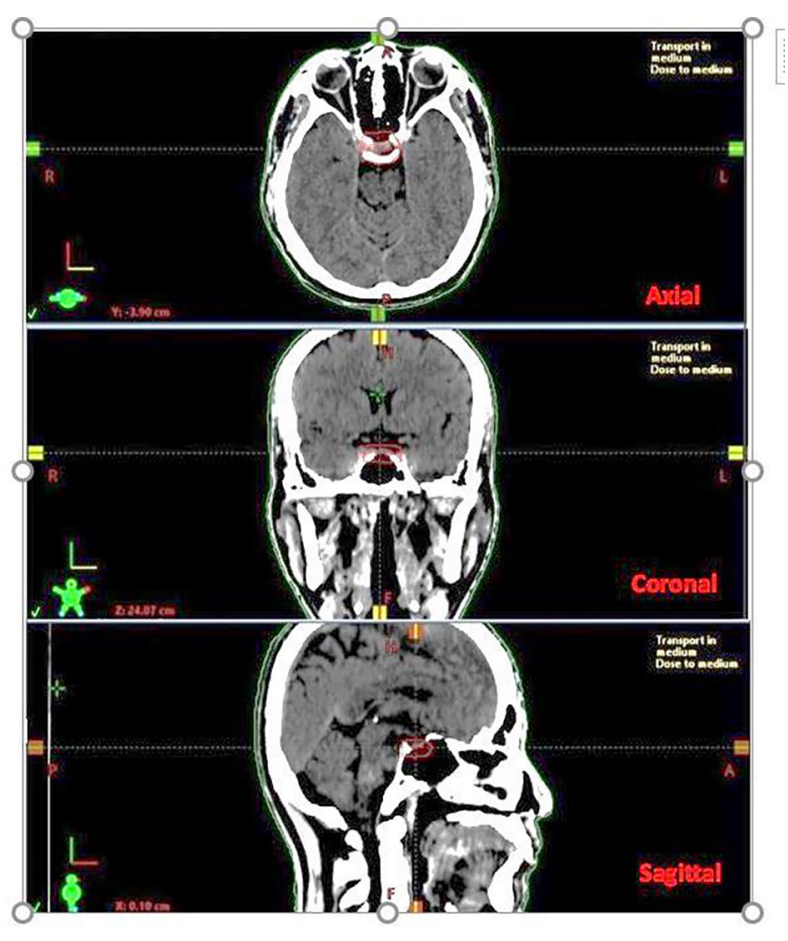

Figure 1 The PTV volume inside the air cavity shown in the red line for axial, coronal and sagittal planes. PTV, planning target volume.

model calculates tissue inhomogeneities anisotropically by using 13 lateral photon scatter kernels. Superposition of the doses determined from photon and electron convolutions gives the final dose distribution. In many cases, the overestimate dose between air and water interface was reported for AAA (2-6). On the other hand, the AXB applies the Linear Boltzmann Transport Equation (LBTE) Solvers in dose calculation modelling method which describes the macroscopic behavior of radiation particles as they travel through and interact with the material (7). Thus, AXB for dose estimation inside and around the inhomogeneity region agrees well with the Monte Carlo (MC) calculation method. AAA is an overestimate dose in the inhomogeneity region compared with MC (4). Moreover, some studies have been published the dose comparison between AAA and $\mathrm{AXB}$, the results shown the dose calculation of AXB was more accurate dose prediction than AAA in inhomogeneity phantom (4,8-11). The aim of AXB is to approve for accurate modeling of the absorb dose in medium.

The dose comparison between the two algorithms has been studied and concerned in past few years. Many publications have evaluated the dose difference of AXB in many regions of carcinoma with AAA calculation. They found the highest dose variation in heterogeneity medium interface between air and tissue. They considered the dose difference impacted in the homogenous target volume only $(9,10)$. Although Zhang et al. (12) published the dose different of AAA and AXB in the lung tumor which it contains low-density tissue, the whole target volume is still homogeneous. The dose coverage of target volume is no statistically significant difference between AAA and AXB. In addition, the effect of dose for heterogeneity as lung and air is difference. From Kim et al. (13) studied the dose comparison in heterogeneity phantom slab between AAA and AXB of about $2.8 \%$ and $20 \%$ in lung tissue and in air, respectively. AAA calculation is more overestimate dose in air than in lung too much that the results agreed with Fogliata et al. (14). So, in the clinical, the cases which contain the air cavity in the treatment field should be concerned the dose in air inhomogeneity They found the dose different between AAA and AXB enhancing in light lung and air material than in normal lung tissue for inhomogeneity phantom.

This study investigated the dose difference impacted in the heterogenous target volume by IMRT and VMAT treatment techniques in a pituitary carcinoma region. When the gross target volume (GTV) is expanded to the planning target volume (PTV), it is commonly overlapping with the nasal air cavity. The PTV has filled in some part by air cavity inside the volume. From the previous studies (4,8-11), the dose difference was more impact in near interface of air and tissue. The dose should be concerned in heterogeneous tissue. Especially, the tumor has filled with the air cavity as shown in Figure 1. It affects with the dose coverage of the target when the plan is evaluated.

Therefore, the objective of this study is to investigate the dose variation between AAA and AXB calculation algorithms in heterogenous inside the PTV of pituitary carcinoma cases. PTV has the air cavity filled inside which effects with the dose distribution and dose coverage in plan. The dose differences between two algorithms were also determined and discussed before using in clinic.

We present the following article in accordance with the MDAR reporting checklist (available at http://dx.doi. org/10.21037/tro-20-57).

\section{Methods}

\section{Treatment planning algorithm}

The Eclipse treatment planning systems was used to all plans 
Table 1 The volumes of PTV and air cavity filled within PTV

\begin{tabular}{lrcc}
\hline Case No. & $\begin{array}{r}\text { PTV volume } \\
\left(\mathrm{cm}^{3}\right)\end{array}$ & $\begin{array}{c}\text { Air cavity volume } \\
\left(\mathrm{cm}^{3}\right)\end{array}$ & $\begin{array}{c}\text { \% air cavity inside } \\
\text { PTV volume }\end{array}$ \\
\hline 1 & 25.20 & 3.24 & 12.86 \\
2 & 4.21 & 1.02 & 24.23 \\
3 & 14.04 & 1.99 & 14.17 \\
4 & 34.55 & 3.10 & 8.97 \\
5 & 35.49 & 4.01 & 11.30 \\
6 & 20.69 & 3.75 & 18.12 \\
7 & 18.00 & 1.20 & 6.67 \\
8 & 20.45 & 2.01 & 9.83 \\
9 & 54.23 & 5.57 & 10.27 \\
10 & 6.36 & 0.32 & 5.03 \\
11 & 16.81 & 0.64 & 3.81 \\
12 & 22.22 & 2.64 & 11.88 \\
\hline
\end{tabular}

PTV, planning target volume.

Table 2 The dose constraints criteria for PTV and normal organs optimization

\begin{tabular}{lc}
\hline Structures & Dose constraints (cGy) \\
\hline PTV & $\mathrm{D}_{95 \%}>5,040$ \\
Brainstem & $\mathrm{D}_{\max }<5,400$ \\
Optic chiasm & $\mathrm{D}_{\max }<5,400$ \\
Optic nerves & $\mathrm{D}_{\max }<5,400$ \\
Cochlear & $\mathrm{D}_{\text {mean }}<4,500$ \\
\hline
\end{tabular}

PTV, planning target volume.

version 15 for $\mathrm{AAA}$ and $\mathrm{AXB}$. $\mathrm{AAA}$ and $\mathrm{AXB}$ were configured in the treatment planning system with the same beam data set. All plans employed the $6 \mathrm{MV}$ photon beam of Varian Clinac TrueBeam machine which was attached with the 120 multileaf collimator (Varian Medical Systems, Polo Alto, CA, USA). The MLC width is $0.5 \mathrm{~cm}$ at the central $20 \mathrm{~cm}$ of field and $1 \mathrm{~cm}$ for the outer $20 \mathrm{~cm}$. Both algorithms were used to calculate dose in 12 pituitary carcinoma cases for intensitymodulated radiotherapy (IMRT) and volumetric modulated arc therapy (VMAT) techniques.

\section{Simulation}

All patients took individually a thermo-plastic head mask (Civco medical solution, Coralville, IA, USA) for immobilization which was attached to a base plate indexed bar on the treatment table. The reference points were marked on the mask. The patients were scanned by computed tomography (CT) simulator (Siemens Somatom definition AS 64-slice, Erlangen, Germany). The 1-mm slice thickness was scanned along the skull vertex to the clavicles. The images were imported to Eclipse treatment planning system. The magnetic resonance imaging (MRI) was registered with CT imaging for target volume delineation.

\section{Target volume and organs at risk (OARs) delineation}

The target volume and OARs of 12 cases were delineated by radiation oncologist. The GTV was expanded to the PTV overlapping with the nasal air cavity. The PTV had filled in some part by air cavity inside the volume. The air cavity has the Hounsfield unit (HU) of $-1,000 \mathrm{HU}$. Target volumes and air cavity volume in each case are shown in Table 1. Brainstem, optic chiasm, optic nerves, and cochleares was contoured as the OARs. The dose constraints and dose prescription are shown in Table 2 according to a radiation oncologist's guide for organ at risk in brain and their dose constraints (15). Conventional fractionation was applied for total dose of 50.4 Gy in 28 fractions, once a day, 5 days per week (16).

\section{Treatment planning}

All IMRT plans were provided using $6 \mathrm{MV}$ photon beam of nine fields around patients. The gantry angle started from $200^{\circ}$ to $160^{\circ}$ with $40^{\circ}$ intervals. The collimator rotation was $0^{\circ}$ for all fields. The dose rate was set at 400 monitor unit $(\mathrm{MU}) / \mathrm{min}$. The smart MLC was selected as MLC sequence mode for leaves motion calculation after the optimization finished.

For VMAT technique, all plans were created with double Arcs for full rotation $\left(360^{\circ}\right)$ with $6 \mathrm{MV}$ photon beam, which started from the counter clockwise arc in the first arc plan. The maximum dose rate was set to $600 \mathrm{MU} / \mathrm{min}$ and then it was controlled automatic by the optimization process. The same dose constraints for optimization with IMRT plans were set.

All plans were calculated by using AAA and AXB. The dose optimization used the photon optimizer (PO) algorithm version 15 in Eclipse treatment planning. Dose correction for tissue heterogeneity was applied. AXB 
Table 3 The average dosimetric comparison of AAA and AXB for both IMRT and VMAT in PTV

\begin{tabular}{|c|c|c|c|c|c|c|}
\hline PTV & \multicolumn{3}{|c|}{ IMRT (n=12) } & \multicolumn{3}{|c|}{ VMAT $(n=12)$} \\
\hline$D_{95}$ (Gy) & $51.1 \pm 0.6$ & $48.9 \pm 3.1$ & 0.028 & $50.2 \pm 1.8$ & $47.6 \pm 4.8$ & 0.020 \\
\hline$D_{98}$ (Gy) & $50.6 \pm 0.7$ & $46.3 \pm 3.7$ & 0.002 & $49.4 \pm 1.9$ & $46.6 \pm 5.3$ & 0.001 \\
\hline $\mathrm{D}_{\max }(\mathrm{Gy})$ & $53.7 \pm 0.5$ & $54.9 \pm 0.9$ & $<0.001$ & $53.9 \pm 1.0$ & $54.7 \pm 1.0$ & $<0.001$ \\
\hline
\end{tabular}

AAA, anisotropic analytical algorithm; AXB, Acuros XB; IMRT, intensity-modulated radiotherapy; VMAT, volumetric modulated arc therapy; PTV, planning target volume; $\mathrm{Cl}$, conformity index.

calculation utilized the same MU and MLC pattern the same as the AAA calculation. The dose calculation grid was set to $2.5 \mathrm{~mm}$ for all cases. For AXB, the dose to medium mode was used for dose calculation. Then, no normalization mode was automatically selected for all plans of IMRT and VMAT calculated by both algorithms. The dose was verified by measurement using ArcCheck diode array detector before all VMAT plans were sent to treat patient. The dose comparison was analyzed by Gamma index (3\%, $3 \mathrm{~mm}$ ) under $95 \%$ passing rate.

For analysis, although the plans used 2 treatment techniques as IMRT and VMAT, the dose comparison between AAA and AXB algorithm was determined in each same treatment technique.

\section{Statistical analysis}

For the PTV, the dose parameters generated by the dosevolume histogram (DVH) using Eclipse treatment planning are conformity index (CI), dose coverage, maximum dose, $\mathrm{D}_{98}$ and $\mathrm{D}_{95}$. The maximum dose $\left(\mathrm{D}_{\max }\right)$ of plan must not be more than $110 \%$ of prescription dose. Therefore, $D_{\max }$ was determined for PTV. The CI was used to evaluate the treatment plans calculated by Equation [1] (17).

$$
C I=\frac{P T V_{P}}{T V}
$$

where $\mathrm{PTV}_{\mathrm{P}}$ is the volume of target receiving the prescription dose and TV is the target volume (17-19). CI is a parameter to explain the good dose conformed in PTV. The perfect conformity value is a unity.

For the OARs, the analytic parameters applied the mean dose and the maximum dose $(1 \mathrm{cc}$ for brainstem and $1 \%$ for the other organs). The results of dose difference between AAA and AXB were compared using $t$-test for statistical analysis. The threshold value was set at $\mathrm{P}<0.05$ for significant difference. The percentage of dose difference (\% dose diff) was computed using Equation [2].

$$
\% \text { Dose diff }=\left(\frac{\text { Dose of } A A A-\text { Dose of } A X B}{\text { Dose of } A X B}\right) \times 100
$$

\section{Ethical statement}

The study was conducted in accordance with the Declaration of Helsinki (as revised in 2013). The study was approved by institutional review board (IRB) of Faculty of Medicine, Chulalongkorn University, Bangkok, Thailand of No. 268/62 and informed consent was taken from all the patients.

\section{Results}

\section{Target volume}

The doses were analyzed with DVH. The average dosimetry results of PTV calculated by AAA and AXB for IMRT and VMAT techniques are shown in Table 3. The example DVHs of PTV comparisons between AAA and AXB calculations for IMRT and VMAT techniques are shown in Figure 2. The dose of PTV was normalize at $95 \%$ of isodose line, for dose coverage of target compared between AAA and AXB calculation. The dosimetric comparisons of PTV between AAA and $\mathrm{AXB}$ at $\mathrm{D}_{95}, \mathrm{D}_{98}$, and maximum dose for each case are shown in Figure 3. The highest differences of dose at $\mathrm{D}_{95}, \mathrm{D}_{98}$, and maximum dose were $21.38 \%, 25.79 \%$ and $5.46 \%$ for IMRT technique, and $27.92 \%, 33.25 \%$ and $2.33 \%$ for VMAT technique, respectively. The dose of AAA was higher than $\mathrm{AXB}$ in all cases at $\mathrm{D}_{95}$ and $\mathrm{D}_{98}$ supported by $\mathrm{P}$ value. On the other hand, the maximum dose of AXB was significantly higher than AAA in all cases. The highest difference in dose was found in case no. 2, which the dose coverage was dropped when it was calculated by the AXB 


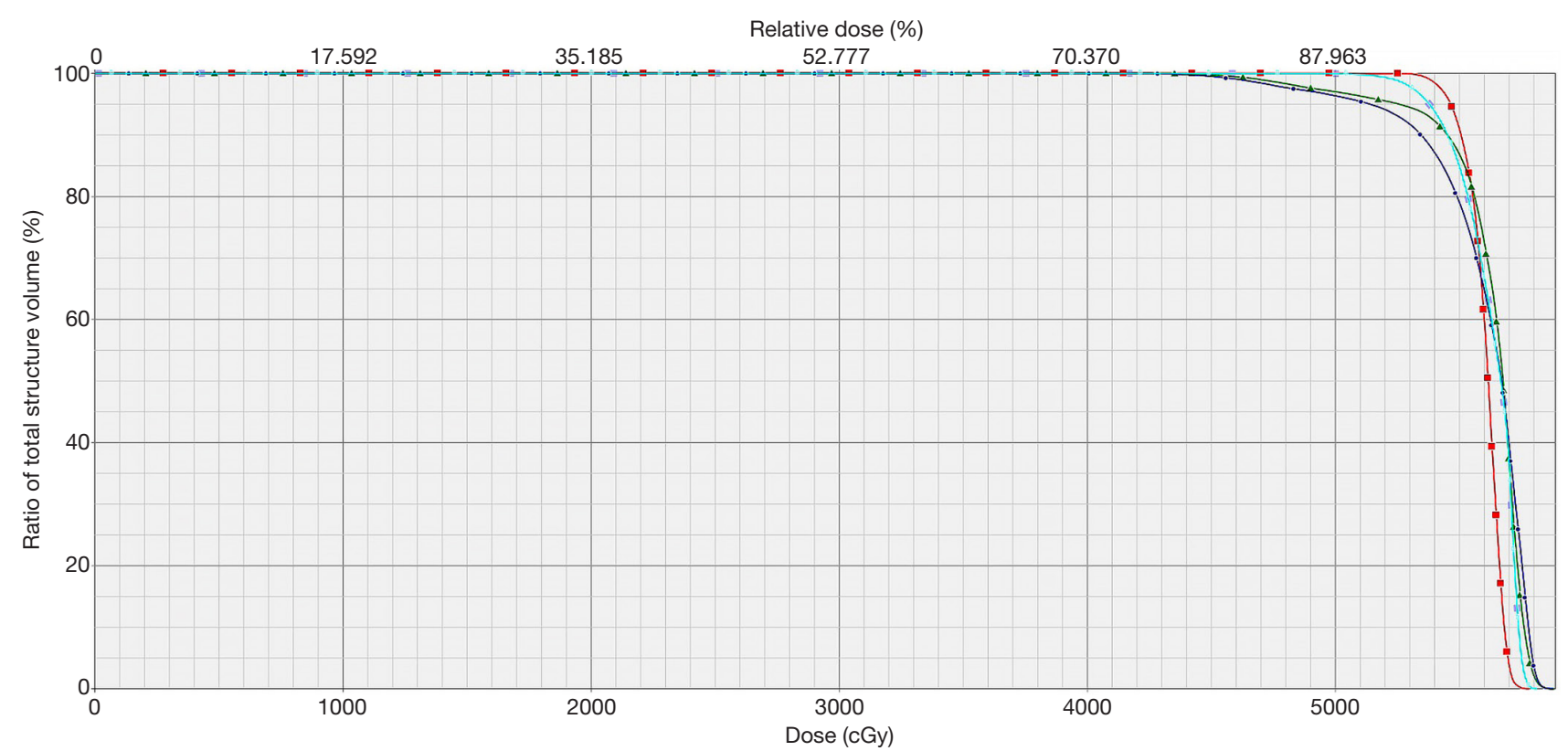

Figure 2 The example DVH of PTV comparisons between AAA and AXB calculations for IMRT and VMAT techniques (red line: AAA for IMRT, Green line: AXB for IMRT, cyan line: AAA for VMAT and blue line: AXB for VMAT). DVH, dose-volume histogram; PTV, planning target volume; AAA, anisotropic analytical algorithm; AXB, Acuros XB; IMRT, intensity-modulated radiotherapy; VMAT, volumetric modulated arc therapy.

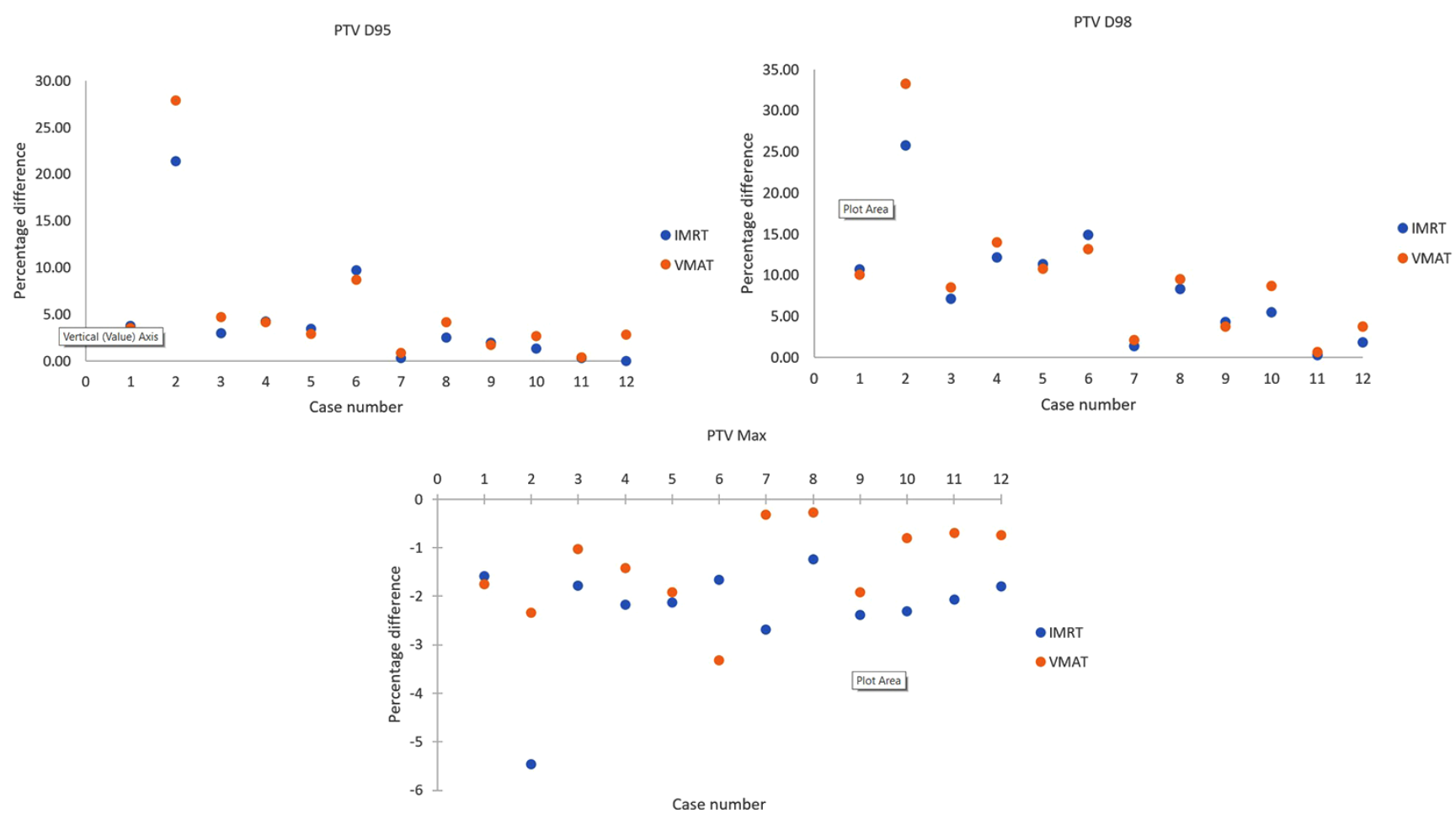

Figure 3 The dose differences between AAA and AXB calculation for both IMRT and VMAT plans for each case of patient. AAA, anisotropic analytical algorithm; AXB, Acuros XB; IMRT, intensity-modulated radiotherapy; VMAT, volumetric modulated arc therapy; PTV, planning target volume. 

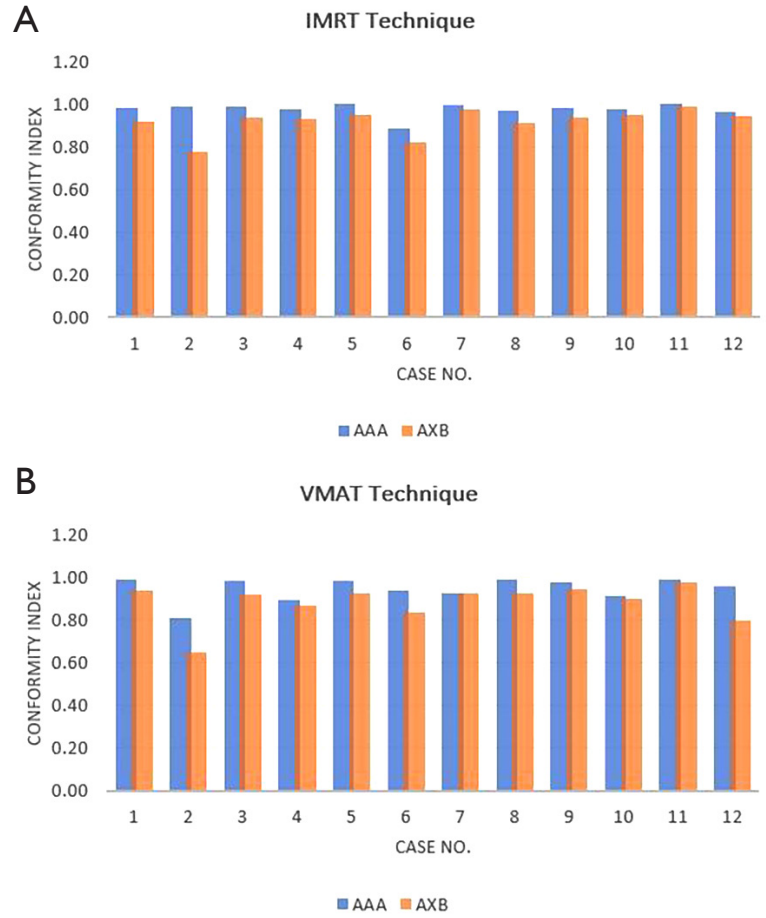

Figure 4 The conformity index value of AAA and AXB. (A) Using IMRT technique. (B) Using VMAT technique. AAA, anisotropic analytical algorithm; AXB, Acuros XB; IMRT, intensity-modulated radiotherapy; VMAT, volumetric modulated arc therapy.

algorithm. This case is a small target volume and a high volume of air cavity filled.

For plan quality evaluation, the CIs for both dose algorithms are presented in Table 3. The CI comparison between two algorithms for both IMRT and VMAT plans are displayed in Figure 4. The CI value of 1.00 represents the perfect coverage of prescribed dose to PTV. The CI decreased when the dose calculation algorithm was changed from AAA to AXB in both IMRT and VMAT treatment techniques. The difference of CI value between AAA and AXB was significant with $\mathrm{P}$ value $<0.05$ in both treatment techniques. So, the dose conformity and dose coverage of AAA was better than AXB in all cases.

The dose distributions of axial plane displayed at the central axis of IMRT and VMAT plans for both calculation algorithms are shown in Figure 5. It was found that isodose line of dose prescription did not cover a whole of PTV, especially in the overlapping area with the air cavity. Therefore, when the AXB was used to calculate in the same plans, the prescribed isodose line was not cover the PTV compared with the AAA for both IMRT and VMAT plans.

\section{Normal organs}

The doses of normal organs were calculated by AAA and AXB for IMRT and VMAT techniques shown in Table 4. The maximum doses as well as $\mathrm{D}_{\text {lcc }}$ for brain stem in IMRT plans between AAA and AXB was 40.6 \pm 5.9 and 40.7 $\pm 5.9 \mathrm{~Gy}$, respectively. On the other hand, the $\mathrm{D}_{\text {lcc }}$ in VMAT plans was $38.8 \pm 6.6$ and $38.7 \pm 6.5$ Gy for AAA and AXB calculations, respectively. For optic chiasm, the maximum doses $\left(\mathrm{D}_{\text {max }}\right)$ between AAA and AXB was 50.8 \pm 2.9 and 51.1 \pm 3.1 Gy for IMRT techniques, while VMAT techniques received the dose of $50.8 \pm 3.1$ and $51.0 \pm 3.2 \mathrm{~Gy}$.

In the case of both optic nerves, for IMRT plans, the $\mathrm{D}_{1 \mathrm{cc}}$ of right optic nerve calculated by AAA and AXB was $34.3 \pm 9.5$ and 34.6 $\pm 9.7 \mathrm{~Gy}$, respectively. For VMAT plans, the right optic nerve received dose of $32.4 \pm 8.6$ and $32.5 \pm 8.7$ Gy for $\mathrm{AAA}$ and AXB algorithms, respectively. For left optic nerve, AAA and AXB algorithms provided the $\mathrm{D}_{1 \mathrm{cc}}$ of $37.1 \pm 10.3$ and $37.3 \pm 10.5$ Gy, respectively, for IMRT plans. For VMAT plans, the left optic nerve received dose of $35.4 \pm 10.2$ and 35.7 \pm 10.3 Gy for AAA and AXB algorithms, respectively. The maximum dose of both cochlear was very less compared with tolerance dose.

For the normal organs, the doses calculated by AAA was slightly less than by AXB in all organs for both IMRT and VMAT techniques. Moreover, mostly normal organs dose by using IMRT technique was higher than by using VMAT technique in the same calculation algorithms. However, the dose of normal organs were not any significant statistic differences in both algorithms and treatment techniques by $\mathrm{P}$ value $>0.05$.

\section{Discussion}

In this study, the determination of dose calculation algorithm AAA against AXB was compared in clinical situation for pituitary cancer cases. The dose comparison between both algorithms was determined in the same treatment technique. This is an interesting case because some area of PTV was filled with the air cavity. The validation was performed by IMRT and VMAT treatment techniques. The dose result calculated by AXB covered the target lesser than AAA in air cavity region for both treatment techniques. It can be analyzed by $\mathrm{D}_{95}, \mathrm{D}_{98}$ and CI values from DVH. So, the doses at $\mathrm{D}_{95}$ and $\mathrm{D}_{98}$ values 

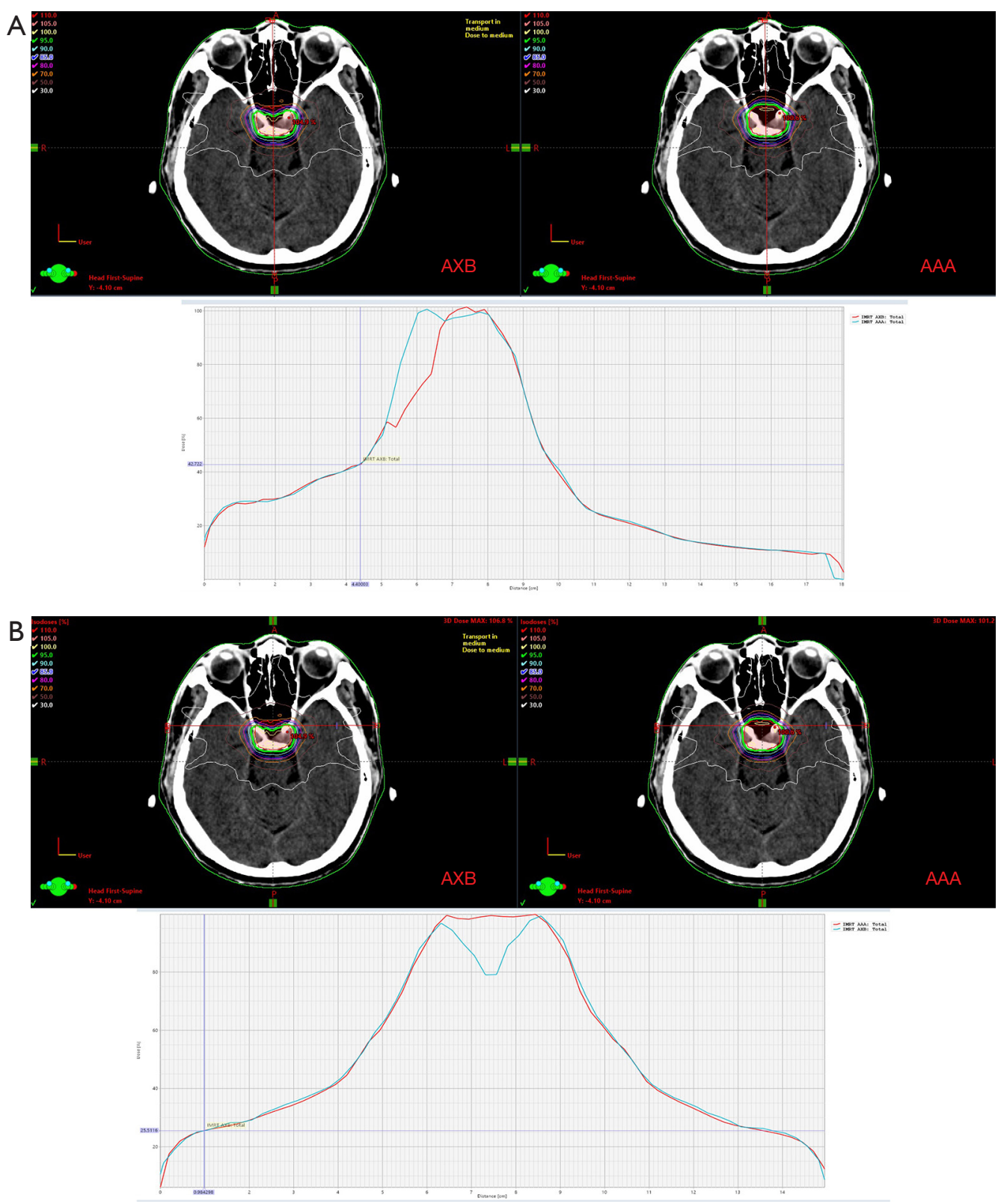

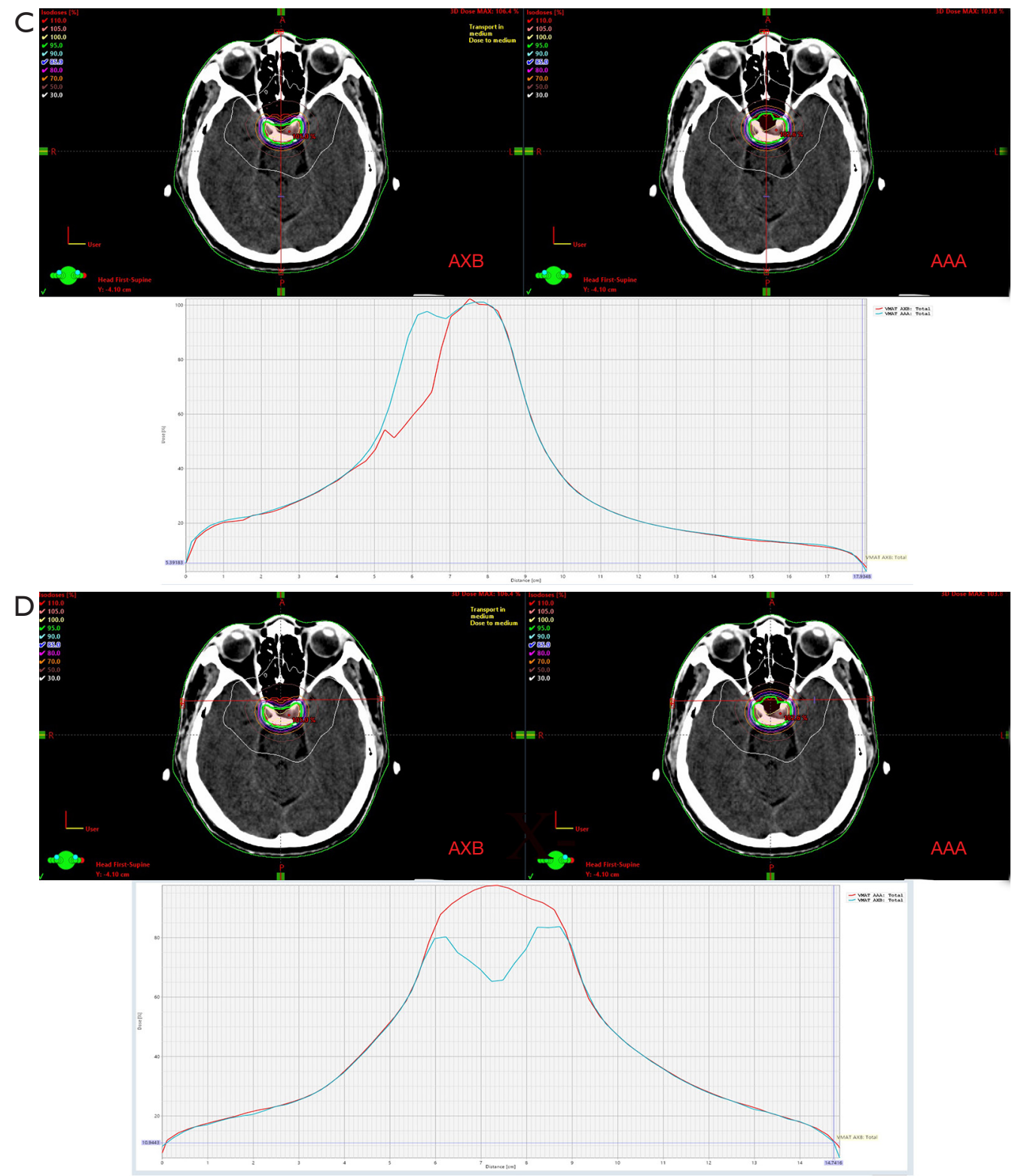

Figure 5 The dose distribution and dose profile of AAA and AXB for IMRT and VMAT plans. For dose profile, blue line is AXB calculation and red line is AAA calculation. (A) The dose distribution of axial plane and the dose profile of anteroposterior axis comparison between AXB and AAA for IMRT plans. (B) The dose distribution of axial plane and the dose profile of right-left lateral axis comparison between AXB and AAA for IMRT plans. (C) The dose distribution of axial plane and the dose profile of anteroposterior axis comparison between AXB and AAA for VMAT plans. (D) The dose distribution of axial plane and the dose profile of right-left lateral axis comparison between AXB and AAA for VMRT plans. AAA, anisotropic analytical algorithm; AXB, Acuros XB; IMRT, intensity-modulated radiotherapy; VMAT, volumetric modulated arc therapy.

from AXB were significantly lower than dose from AAA. Moreover, CI values of AXB was lower than of AAA for both treatment techniques, the different values were significant. When the dose was determined by visual isodose distribution, the prescribed dose was dropped in the cavity region. Especially, in the case of a small target volume and a high volume of air cavity filled, the dose difference was too high such as the case number 2. This effect caused 
Table 4 The average dosimetric comparison of AAA and AXB for both IMRT and VMAT techniques in normal organs

\begin{tabular}{|c|c|c|c|c|}
\hline Organ at risk & \multicolumn{2}{|c|}{ IMRT (n=12) } & \multicolumn{2}{|c|}{ VMAT $(n=12)$} \\
\hline \multicolumn{5}{|l|}{ Brain stem } \\
\hline$D_{\text {mean }}(G y)$ & $18.2 \pm 5.8$ & $18.3 \pm 5.8$ & $17.1 \pm 6.2$ & $17.2 \pm 6.2$ \\
\hline$D_{1 c c}(G y)$ & $40.6 \pm 5.9$ & $40.7 \pm 5.9$ & $38.7 \pm 6.1$ & $38.8 \pm 6.1$ \\
\hline $\mathrm{D}_{\text {mean }}(\mathrm{Gy})$ & $44.3 \pm 7.8$ & $44.6 \pm 7.9$ & $44.1 \pm 7.2$ & $44.3 \pm 7.5$ \\
\hline$D_{\max }(G y)$ & $50.8 \pm 2.9$ & $51.1 \pm 3.1$ & $50.8 \pm 3.1$ & $51.0 \pm 3.2$ \\
\hline \multicolumn{5}{|c|}{ Right optic nerve } \\
\hline $\mathrm{D}_{\text {mean }}(\mathrm{Gy})$ & $17.3 \pm 5.5$ & $17.5 \pm 5.6$ & $17.1 \pm 5.3$ & $17.2 \pm 5.2$ \\
\hline $\mathrm{D}_{\text {mean }}(\mathrm{Gy})$ & $19.2 \pm 6.5$ & $19.3 \pm 6.5$ & $19.9 \pm 6.6$ & $20.0 \pm 6.7$ \\
\hline$D_{\max }(G y)$ & $35.7 \pm 8.6$ & $35.8 \pm 8.7$ & $34.1 \pm 8.6$ & $34.4 \pm 8.6$ \\
\hline \multicolumn{5}{|l|}{ Right cochlear } \\
\hline$D_{\text {mean }}(G y)$ & $10.7 \pm 5.4$ & $10.9 \pm 5.7$ & $10.0 \pm 5.6$ & $10.2 \pm 5.8$ \\
\hline $\mathrm{D}_{\max }(\mathrm{Gy})$ & $15.6 \pm 6.4$ & $16.0 \pm 6.6$ & $14.5 \pm 6.4$ & $14.8 \pm 6.7$ \\
\hline \multicolumn{5}{|l|}{ Left cochlear } \\
\hline $\mathrm{D}_{\text {mean }}(\mathrm{Gy})$ & $10.8 \pm 5.1$ & $10.9 \pm 5.2$ & $10.1 \pm 5.2$ & $10.3 \pm 5.4$ \\
\hline $\mathrm{D}_{\max }(\mathrm{Gy})$ & $17.2 \pm 8.4$ & $17.6 \pm 8.6$ & $16.7 \pm 9.7$ & $17.0 \pm 9.7$ \\
\hline
\end{tabular}

AAA, anisotropic analytical algorithm; AXB, Acuros XB; IMRT, intensity-modulated radiotherapy; VMAT, volumetric modulated arc therapy.

the AAA overestimated dose in air or low density due to lack of scattering when compared with $\operatorname{AXB}(11,14,20)$. Accordingly, AXB algorithm was more accurate than AAA from calculated dose in heterogeneity medium $(3,8,9,11,14)$. Many studies reported the dose comparison of AAA and AXB with MC simulation. They found that the dose of $\mathrm{AXB}$ agreed with MC better than the dose of AAA for inhomogeneous medium $(14,21)$.

The dose differences between AAA and AXB were larger in the very low-density region for small field size and large air gap. Fogliata et al. (14) studied the dose difference of AAA and AXB comparison with MC in low density (normal lung of $-790 \mathrm{HU}$ ) and very low density (light lung of $-942 \mathrm{HU}$ ). They found the dose difference of AAA and MC in very low density higher than low density. On the other hand, the dose difference of $\mathrm{AXB}$ and MC was good agreement in both tissue densities. Inside the most internal light lung material the differences between $\mathrm{AXB}$ and MC were small. So, The HU affected with AAA more than AXB algorithms in low density. The HU of air cavity filled in this study was $-1,000 \mathrm{HU}$. Rana et al. (6) showed the dose differences between calculation algorithms and measurement. The differences were larger in small field sizes for both AAA and AXB. These effects were enhanced for AAA than AXB. For dose comparison between different air gap distance, the dose difference was higher when the air gap was thicker. There are the reasons for the dose differences between AAA and AXB enhanced in the case that had the large air cavity filled and a small volume of PTV. Thus, it affects to dose coverage of the target in this study according to CI value. From the results, the CI values calculated by AXB were less than calculated by AAA for all cases. The comparison of dose coverage between AAA and AXB showed a statistical difference for both IMRT and VMAT plans by $\mathrm{P}$ value. Although AAA seems better than AXB for dose coverage of PTV, AXB is more accurate 
than AAA for dose calculation in inhomogeneous medium. Thus, the dose which shows the good coverage of AAA is incorrect in the air cavity region. The dose predicted by AAA and AXB depends on the combined effects of field size, the thickness of the air cavity, and photon energy $(6,14)$. However, this study did not investigate the different energy.

Normal organs dose was similar for both AAA and $\mathrm{AXB}$, and for all treatment techniques. The dose difference in normal organs did not show any statistical difference because the dose calculated by AAA and AXB was good agreement in homogeneous tissue (9). The doses calculated by AAA was less than by AXB in all organs.

\section{Conclusions}

The dose was compared in air cavity region of target volume for pituitary carcinoma by AAA and AXB algorithms. The dose coverage of target using AXB was less than using AAA for calculation in the air cavity region which it depended on the size of target and volume of air cavity. In general, the $\mathrm{AXB}$ can calculate dose accurately than AAA for inhomogeneous region. The good dose coverage to target displayed by AAA calculation should be determined carefully in clinical when evaluation dose in the target is filled by air cavity. To be able to accept the best plan in normal practice. The evaluation parameters such as $\mathrm{D}_{95}$, $\mathrm{D}_{98}$, Dmax and CI for target volume, and dose tolerance for normal tissues must be used to determine. Together with other tools (DVH, isodose distribution etc.), it should be determined within the accepted criteria. An attempt should be made to avoid large air gaps when smaller field sizes are used for the radiation treatment.

\section{Acknowledgments}

This study was supported by medical physicist of King Chulalongkorn Memorial Hospital, Division of Therapeutic Radiology and Oncology.

Funding: None.

\section{Footnote}

Reporting Checklist: The authors have completed the MDAR reporting checklist. Available at http://dx.doi.org/10.21037/ tro-20-57

Conflicts of Interest: All authors have completed the ICMJE uniform disclosure form (available at http://dx.doi. org/10.21037/tro-20-57). The authors have no conflicts of interest to declare.

Etbical Statement: The authors are accountable for all aspects of the work in ensuring that questions related to the accuracy or integrity of any part of the work are appropriately investigated and resolved. The study was conducted in accordance with the Declaration of Helsinki (as revised in 2013). The study was approved by institutional review board (IRB) of Faculty of Medicine, Chulalongkorn University, Bangkok, Thailand of No. 268/62 and informed consent was taken from all the patients.

Open Access Statement: This is an Open Access article distributed in accordance with the Creative Commons Attribution-NonCommercial-NoDerivs 4.0 International License (CC BY-NC-ND 4.0), which permits the noncommercial replication and distribution of the article with the strict proviso that no changes or edits are made and the original work is properly cited (including links to both the formal publication through the relevant DOI and the license). See: https://creativecommons.org/licenses/by-nc-nd/4.0/.

\section{References}

1. Report 24. Journal of the International Commission on Radiation Units and Measurements 1976;os13. Available online: https://doi.org/10.1093/jicru/os13.1.Report24.

2. Alhakeem EA, AlShaikh S, Rosenfeld AB, et al. Comparative evaluation of modern dosimetry techniques near low- and high-density heterogeneities. J Appl Clin Med Phys 2015;16:142-58.

3. Yan C, Combine AG, Bednarz G, et al. Clinical implementation and evaluation of the Acuros dose calculation algorithm. J Appl Clin Med Phys 2017;18:195-209.

4. Zaman A, Kakakhel MB, Hussain A. A comparison of Monte Carlo, anisotropic analytical algorithm (AAA) and Acuros XB algorithms in assessing dosimetric perturbations during enhanced dynamic wedged radiotherapy deliveries in heterogeneous media. J Radiother Pract 2019;18:75-81.

5. Mandal A, Ram C, Mourya A,et al. Small field depth dose profile of $6 \mathrm{MV}$ photon beam in a simple air-water heterogeneity combination: A comparison between anisotropic analytical algorithm dose estimation with thermoluminescent dosimeter dose measurement. J Cancer Res Ther 2017;13:491-7.

6. Rana S, Rogers K. Dosimetric evaluation of Acuros 
$\mathrm{XB}$ dose calculation algorithm with measurements in predicting doses beyond different air gap thickness for smaller and larger field sizes. J Med Phys 2013;38:9-14.

7. Vassiliev ON, Wareing TA, McGhee J, et al. Validation of a new grid-based Boltzmann equation solver for dose calculation in radiotherapy with photon beams. Phys Med Biol 2010;5 5:581-98.

8. Chopra KL, Leo P, Kabat C, et al. Evaluation of dose calculation accuracy of treatment planning systems in the presence of tissue heterogeneities. Ther Radiol Oncol 2018;2:28.

9. Huang B, Wu L, Lin P, et al. Dose calculation of Acuros $\mathrm{XB}$ and Anisotropic Analytical Algorithm in lung stereotactic body radiotherapy treatment with flattening filter free beams and the potential role of calculation grid size. Radiat Oncol 2015;10:53.

10. Padmanaban S, Warren S, Walsh A, et al. Comparison of Acuros (AXB) and Anisotropic Analytical Algorithm (AAA) for dose calculation in treatment of oesophageal cancer: effects on modelling tumour control probability. Radiat Oncol 2014;9:286.

11. Woon YL, Heng SP, Wong JHD, et al., editors. Comparison of selected dose calculation algorithms in radiotherapy treatment planning for tissues with inhomogeneities. J Phys Conf Ser 2016;694:012024.

12. Zhang J, Jiang D, Su H, et al. Dosimetric comparison of different algorithms in stereotactic body radiation therapy (SBRT) plan for non-small cell lung cancer (NSCLC). Onco Targets Ther 2019;12:6385-91.

13. Kim YL, Suh TS, Choe BY, et al. Dose distribution evaluation of various dose calculation algorithms in

doi: $10.21037 /$ tro-20-57

Cite this article as: Israngkul-Na-Ayuthaya I, Suriyapee S, Sanghamthum T. The dosimetric comparison using anisotropic analytical algorithm (AAA) and Acuros XB algorithms in the target overlapping with air cavity of pituitary carcinoma. Ther Radiol Oncol 2021;5:7. inhomogeneous media. Int J Radiat Res 2016;14:269-78.

14. Fogliata A, Nicolini G, Clivio A, et al. Dosimetric evaluation of Acuros XB Advanced Dose Calculation algorithm in heterogeneous media. Radiat Oncol 2011;6:82.

15. Scoccianti S, Detti B, Gadda D, et al. Organs at risk in the brain and their dose-constraints in adults and in children: a radiation oncologist's guide for delineation in everyday practice. Radiother Oncol 2015;114:230-8.

16. Kosmin M, Fersht N. Radiotherapy for Pituitary Tumors. In: Feingold KR, Anawalt B, Boyce A, et al., editors. Endotext [Internet]. South Dartmouth (MA): MDText. com, Inc., 2017.

17. Huchet A, Caudry M, Belkacémi Y, et al. Volume-effect and radiotherapy [II]. Part II: volume-effect and normal tissue. Cancer Radiother 2003;7:353-62.

18. Feuvret L, Noël G, Mazeron JJ, et al. Conformity index: a review. Int J Radiat Oncol Biol Phys 2006;64:333-42.

19. Shaw E, Scott C, Souhami L, et al. Single dose radiosurgical treatment of recurrent previously irradiated primary brain tumors and brain metastases: final report of RTOG protocol 90-05. Int J Radiat Oncol Biol Phys 2000;47:291-8.

20. Israngkul $\mathrm{Na}$ Ayuthaya I, Suriyapee S, Sanghangthum T, et al. Dose Evaluation of Treatment Planning System in Inhomogeneous Media. Australasian Physical Engineering Sciences in Medicine 2007;30:488-9.

21. Schneider W, Bortfeld T, Schlegel W. Correlation between CT numbers and tissue parameters needed for Monte Carlo simulations of clinical dose distributions. Phys Med Biol 2000;45:459-78. 